\title{
SOME REMARKS ON FACTORIZATION OF ENTIRE FUNCTIONS
}

\author{
Dedicated to Professor N. Suita for his 60-th birthday
}

\author{
By HiRONOBU URABE
}

\begin{abstract}
In connection with the results of G. Song and J. Huang as well as G. Song and L. Liao, concerning the factorization of entire functions, we shall prove the existence of another entire function which is prime but whose square is not pseudo-prime, by making use of Y. Noda's argument.
\end{abstract}

\section{Introduction.}

A meromorphic function $F(z)=f(g(z))$ is said to have $f$ and $g$ as left and right factors respectively, provided that $f$ is meromorphic and $g$ is entire ( $g$ may be meromorphic when $f$ is rational). Then, $F$ is called to be prime (pseudo-prime) if every factorization of the above form into factors implies that either $f$ or $g$ is linear (either $f$ is rational or $g$ is a polynomial) (cf. [3]).

Recently, G. Song and J. Huang proved the following theorems A and B in [11]. Theorem A. If $f$ is a pseudo-prime entire function and $n$ is an odd integer $(n \geqq 1)$, then $F(z) \equiv\{f(z)\}^{n}$ is also pseudo-prime. Theorem B. The function $F(z) \equiv \sin z \cdot \exp [\cos z]$ is prime.

It is noteworthy that, for the above prime function $F(z)=\sin z \cdot \exp [\cos z]$, $\{F(z)\}^{2}$ is not pseudo-prime: $\{F(z)\}^{2}=(G(w)) \circ(\cos z)$, where $G(w)=\left(1-w^{2}\right)$. $\exp [2 w]$. Moreover, the even power $\{F(z)\}^{2 k}$ ( $k$; a natural number) is not pseudo-prime either, since $\{F(z)\}^{2 k}=\left(G(w)^{k}\right) \circ(\cos z)$. In this case, $F$ is of infinite order.

When the order (of such $F$ ) is finite, G. Song and L. Liao showed a similar result in [12].

In this note, by applying the argument due to Y. Noda ([7]), we shall exhibit other entire functions having the same property as above (that is, entire functions which are prime but whose square and hence even powers are not pseudo-prime).

Received June 18, 1992. 


\section{Results.}

THEOREM 1. Let $h$ be a non-constant entire function such that $h(w)=$ $\varphi\left(\left(2 w^{2}-1\right)^{2}\right)$ with $\varphi(\zeta)=\exp [\psi(\zeta)]$ for some entire function $\phi$ and let $H(z)=$ $\sin z \cdot h(\cos z)$. Then the set

$$
\left\{a \in C ; F_{a}(z) \equiv H(z)-\frac{a}{2} \sin 2 z \text { is not prime }\right\}
$$

is at most countable.

Let us note that the function in the above Theorem 1 can be expressed as $F_{a}(z)=\sin z \cdot\{h(\cos z)-a \cos z\}$. Hence $\left\{F_{a}(z)\right\}^{2}=G_{a}(\cos z)$ with $G_{a}(w)=$ $\left(1-w^{2}\right)\{h(w)-a w\}^{2}$. Thus $\left\{F_{a}(z)\right\}^{2}$ is not pseudo-prime (transcendentally composite).

THEOREM 2. Let $h$ be a non-constant entrre function and $H(z)=\sin z \cdot h(\cos z)$. Suppose that the order of $H$ is finite and not an integer and $H$ has only simple zeros. Then the set

$$
\left\{a \in C ; F_{a}(z) \equiv H(z)(\cos z-a) \text { is not prime }\right\}
$$

is at most countable.

For the function $F_{a}$ in the above Theorem 2, it follows that $\left\{F_{a}(z)\right\}^{2}=$ $\left(\left(1-w^{2}\right) \cdot[h(w)(w-a)]^{2}\right) \circ(\cos z)$.

In order to prove these results, we shall follow the argument due to $Y$. Noda in [7] honestly (see $\S \S 3$ and 4).

3. Lemmas. For the proof of Theorems 1 and 2, we shall need the following lemmas which are the modified versions of Y. Noda's lemma (Lemma 3 in [7]).

LEMMA 1. Let $H(z)=\sin z \cdot h(\cos z)$, where $h(w)=\varphi\left(\left(2 w^{2}-1\right)^{2}\right)$ for some nonconstant entire function $\varphi$. Let $F_{a}(z)=H(z)-(a / 2) \sin 2 z$. Suppose now that $\cos 2 z$ and $H^{\prime}(z)$ have no common zeros. Then, there exists a countable set $E$ of complex numbers such that any two roots $u$ and $v$ of the simultaneous equations

$$
F_{a}(z)=c \text { and } F_{a}{ }^{\prime}(z)=0
$$

satisfy $\cos u=\cos v$ and $\sin u=\sin v$ for any constant $c(\in \boldsymbol{C})$ provided $a \in \boldsymbol{C}-E$.

LEMMA 2. Let $H(z)=\sin z \cdot h(\cos z)$ for a non-constant entire function $h$. Let $F_{a}(z)=H(z)(\cos z-a)$. Suppose that $H(z)$ has only simple zeros. Then, there exists a countable set $E$ of complex numbers such that any two roots $u$ and $v$ of 
the simultaneous equations

$$
F_{a}(z)=c \text { and } F_{a}{ }^{\prime}(z)=0
$$

satısfy $\cos u=\cos v$ and $\sin u=\sin v$ for any constant $c(\in C)$ provided $a \in C-E$.

Proof of Lemma 1. By the assumption, $F_{a}(z)=H(z)-(a / 2) \sin 2 z$ and hence $F_{a}{ }^{\prime}(z)=H^{\prime}(z)-a \cos 2 z$. Let us write

and

$$
k(z)=H^{\prime}(z) / \cos 2 z
$$

$$
A=C-\left\{p \in C ; p \text { is a zero or pole of } k^{\prime}(z)\right\} .
$$

We choose open covering sets $\left\{C_{i}\right\}_{l=1}^{\infty}$ of $A$ satisfying the following conditions.

(1) $\bigcup_{\imath=1}^{\infty} C_{\imath}=A$,

(2) $k(z)$ is univalent in $C_{\imath}(i=1,2, \cdots)$,

(3) $\left\{k(z) ; z \in C_{i}\right\}$ is a disk $(i=1,2, \cdots)$.

We set

$$
\begin{gathered}
D_{\imath}=\left\{k(z) ; z \in C_{i}\right\} \quad(i=1,2, \cdots), \\
K(z)=H(z)-\frac{k(z)}{2} \sin 2 z, \\
x_{i}(w)=\left(\left.k\right|_{C_{i}}\right)^{-1}(w), \quad y_{i}(w)=K\left(x_{i}(w)\right) \quad\left(w \in D_{\imath}, i=1,2, \cdots\right), \\
I=\left\{(i, j) \in \boldsymbol{N} \times \boldsymbol{N} ; D_{i} \cap D_{\jmath} \neq \varnothing, y_{i}(w) \not \equiv y_{j}(w)\left(w \in D_{i} \cap D_{j}\right)\right\}, \\
S_{\imath \jmath}=\left\{w \in D_{i} \cap D_{\jmath} ; y_{i}(w)=y_{j}(w)\right\} \quad((i, j) \in I), \\
E_{0}=\left(\bigcup_{\imath=1}^{\infty} D_{\imath}\right)-\left(\left\{k(p) ; k^{\prime}(p)=0, p \in \boldsymbol{C}\right\} \cup\left(\bigcup_{(i, j) \in I} S_{\imath \jmath}\right)\right. \\
\left.\cup\left(\bigcup_{\imath=1}^{\infty}\left\{p \in D_{\imath} ; H\left(x_{i}(p)\right)=0\right\}\right)\right)
\end{gathered}
$$

and $E=C-E_{0}$.

Then $E$ is a countable set. For this set $E$, we shall verify the validity of the assertion of Lemma 1 as follows. Now, by virtue of the definition,

$$
K^{\prime}(z)=H^{\prime}(z)-\frac{k^{\prime}(z)}{2} \sin 2 z-k(z) \cos 2 z=-\frac{k^{\prime}(z)}{2} \sin 2 z .
$$

If $w \in D_{\imath}(i=1,2, \cdots)$, then $k\left(x_{i}(w)\right)=w$ and hence we have

$$
y_{i}(w)=H\left(x_{i}(w)\right)-w / 2 \cdot \sin \left(2 x_{i}(w)\right)
$$

and also (since $k^{\prime}\left(x_{i}(w)\right) \cdot x_{\imath}{ }^{\prime}(w)=1$ )

$$
y_{\imath}{ }^{\prime}(w)=K^{\prime}\left(x_{i}(w)\right) \cdot x_{\imath}{ }^{\prime}(w)=-\sin \left(2 x_{i}(w)\right) / 2 .
$$


Assume now that $a \in E_{0}(=C-E)$. Then the set

$$
\left\{x_{i}(a) ; a \in D_{\imath}(i=1,2, \cdots)\right\}
$$

coincides with the set of the roots of $F_{a}{ }^{\prime}(z)=0$. This can be verified as follows. Let us note that, by the assumption, $\cos 2 z$ and $H^{\prime}(z)$ have no common zeros. Then, since $k\left(x_{i}(a)\right)=a$ for $a \in D_{\imath}$, we have $\left(\cos 2 x_{i}(a) \neq 0\right.$ and hence) $F_{a}{ }^{\prime}\left(x_{i}(a)\right)$ $=0$. Conversely, if $F_{a}{ }^{\prime}(s)=H^{\prime}(s)-a \cos 2 s=0$, then $k(s)=a$ (noting $\cos 2 s \neq 0$ ). Since $a \in E_{0}, k^{\prime}(s) \neq 0$ so that $s=x_{i}(a)$ with $a \in D_{\imath}$ for some $i$.

Moreover, if $y_{i}(a)=y_{j}(a)$ for some $a \in E_{0}$, then $y_{i}(w) \equiv y_{j}(w)$ near $w=a$. Hence $y_{\imath}{ }^{\prime}(a)=y_{j}{ }^{\prime}(a)$ as well. Accordingly,

$$
\sin 2 x_{i}(a)=\sin 2 x_{j}(a) \text { and } H\left(x_{i}(a)\right)=H\left(x_{j}(a)\right)
$$

$\left(\neq 0\right.$, since $\left.a \in E_{0}\right)$. The latter equation implies

$$
\sin x_{i}(a) \cdot \varphi\left(1-\sin ^{2}\left[2 x_{i}(a)\right]\right)=\sin x_{j}(a) \cdot \varphi\left(1-\sin ^{2}\left[2 x_{j}(a)\right]\right) .
$$

Hence, in view of the former equation, we obtain $\sin x_{i}(a)=\sin x_{j}(a)(\neq 0)$ and further $\cos x_{i}(a)=\cos x_{j}(a)$ (whence we see $x_{i}(a)-x_{j}(a)=2 n \pi$ for some integer $n)$.

Thus we have the desired rseults.

Proof of Lemma 2. Since $F_{a}(z)=H(z)(\cos z-a)$,

$$
F_{a}^{\prime}(z)=H^{\prime}(z)(\cos z-a)-H(z) \cdot \sin z .
$$

We set

$$
k(z)=\cos z-H(z) \cdot \sin z / H^{\prime}(z) .
$$

By the assumption that $H(z)=\sin z \cdot h(\cos z)$ has only simple zeros, $H^{\prime}(z)$ and $\sin z$ (as well as $H^{\prime}(z)$ and $H(z)$ ) have no common zeros. Therefore, $F_{a}{ }^{\prime}(z)=0$ if and only if $k(z)=a$.

Let us write

$$
A=C-\left\{p \in C ; p \text { is a zero or pole of } k^{\prime}(z)\right\} .
$$

We choose open covering sets $\left\{C_{i}\right\}_{i=1}^{\infty}$ of $A$ (as before) satisfying the following conditions

(1) $\bigcup_{i=1}^{\infty} C_{i}=A$,

(2) $k(z)$ is univalent in $C_{\imath}(i=1,2, \cdots)$,

(3) $\left\{k(z) ; z \in C_{i}\right\} \equiv D_{\imath}$ is a disk $(i=1,2, \cdots)$.

We also set

$$
\begin{gathered}
K(z)=H(z)(\cos z-k(z)), \\
x_{i}(w)=\left(\left.k\right|_{C_{i}}\right)^{-1}(w) \quad\left(w \in D_{\imath}, i=1,2, \cdots\right), \\
y_{i}(w)=K\left(x_{i}(w)\right) \quad\left(w \in D_{\imath}, i=1,2, \cdots\right),
\end{gathered}
$$




$$
\begin{gathered}
I=\left\{(i, j) \in \boldsymbol{N} \times \boldsymbol{N} ; D_{i} \cap D_{j} \neq \varnothing \text { and } y_{i}(w) \neq \equiv y_{j}(w)\left(w \in D_{i} \cap D_{j}\right)\right\}, \\
S_{\imath \jmath}=\left\{w \in D_{i} \cap D_{\jmath} ; y_{i}(w)=y_{j}(w)\right\} \quad((i, j) \in I), \\
E_{0}=\left(\bigcup_{i=1}^{\infty} D_{\imath}\right)-\left(\left\{k(p) ; k^{\prime}(p)=0, p \in \boldsymbol{C}\right\} \cup\left(\bigcup_{(i, j) \in I} S_{\imath \jmath}\right)\right. \\
\left.\cup\left(\bigcup_{\imath=1}^{\infty}\left\{p \in D_{i} ; H\left(x_{i}(p)\right)=0\right\}\right)\right) .
\end{gathered}
$$

Then we have

1). $E \equiv C-E_{0}$ is a countable set,

2). $y_{i}(w)=H\left(x_{i}(w)\right)\left(\cos x_{i}(w)-w\right)$ and $y_{i}{ }^{\prime}(w)=-H\left(x_{i}(w)\right)$ if $w \in D_{\imath}$ for some $i$.

$3)$. If $y_{i}(a)=y_{j}(a)$ for some $a \in E_{0}$, then $\cos x_{i}(a)=\cos x_{j}(a)$ and $\sin x_{i}(a)$ $=\sin x_{j}(a)$.

4). If $a \in E_{0}$, then the set $\left\{x_{i}(a) ; a \in D_{\imath}(i=1,2, \cdots)\right\}$ is identical with the set of the roots of $F_{a}{ }^{\prime}(z)=0$.

In fact, 1) is clearly valid. 2). The former equation is readily seen. Since $K^{\prime}(z)=-k^{\prime}(z) H(z)$, we get $y_{i}^{\prime}(w)=-H\left(x_{i}(w)\right)$ as well. 3). If $y_{i}(a)=y_{j}(a)$ for some $a \in E_{0}$, then $y_{i}(w) \equiv y_{j}(w)$ near $w=a$. Hence $y_{i}{ }^{\prime}(a)=y_{j}^{\prime}(a)$. Hence $H\left(x_{i}(a)\right)\left(\cos x_{i}(a)-a\right)=H\left(x_{j}(a)\right)\left(\cos x_{j}(a)-a\right)$ and $H\left(x_{i}(a)\right)=H\left(x_{j}(a)\right)(\neq 0$, since $\left.a \in E_{0}\right)$. Then $\cos x_{\imath}(a)=\cos x_{j}(a)$. Moreover, by the latter equation, it follows that $\sin x_{i}(a) \cdot h\left(\cos x_{i}(a)\right)=\sin x_{j}(a) \cdot h\left(\cos x_{j}(a)\right)(\neq 0)$ and so that $\sin x_{i}(a)=$ $\sin x_{j}(a)$ at the same time. 4). Noting the fact that $F_{a}{ }^{\prime}(z)=0$ if and only if $k(z)=a$, we can easily verify the assertion, since, if $a \in E_{0}, k(s)=a$ if and only if $s=x_{i}(a)$ with $a \in D_{\imath}$ for some $i$.

Hence, we also have the desired result.

\section{Proof of Theorems.}

Proof of Theorem 1. We have $F_{a}(z)=H(z)-a / 2 \cdot \sin 2 z$ and $H(z)=\sin z$. $h(\cos z)$, where $h(w)=\varphi\left(\left(2 w^{2}-1\right)^{2}\right)$ with $\varphi(\zeta)=\exp [\psi(\zeta)]$ for some non-constant entire function $\phi$. As being shown in the previous section, we can choose a countable set $E$ of complex numbers for which the assertion of Lemma 1 holds with respect to the above $F_{a}(z)$. We (may) assume $0 \in E$.

In what follows, we shall assume that $a \in E_{0}=C-E$ and prove that $F_{a}(z)$ is indeed prime, by applying Noda's argument in [7] (cf. especially the argument in the proof of Theorem 3, there).

Let $t \in(0,1)$. Then (since $a \neq 0$ ), the inequalities

and

$$
\bar{N}\left(r, 0, F_{a}^{\prime}\right) \geqq \operatorname{tm}\left(r, H^{\prime}\right)
$$

$$
\bar{N}\left(r, c, F_{a}\right) \geqq \operatorname{tm}(r, H)
$$

hold (respectively) on a set of $r$ of infinite measure for any complex number $c$, 
which is seen to be valid by the modified second fundamental theorem using small functions (cf. Theorem 2.5 in [4], p. 47) and also Clunie's theorem ([1]). Here we used Nevanlinna's notations ([6]).

Letting $F_{a}(z)=f(g(z))$, we consider the following several cases separately one by one.

a). $f$ and $g$ are transcendental entire functions. Since $F_{a}{ }^{\prime}(z)=f^{\prime}(g(z)) g^{\prime}(z)$, we see $f^{\prime}(w)$ has infinitely many zeros $\left\{w_{n}\right\}_{n=1}^{\infty}$ (say) by the inequality (*). Then, any root of $g(z)=w_{n}$ is also a common root of the simultaneous equations

$$
F_{a}(z)=f\left(w_{n}\right) \text { and } \quad F_{a}^{\prime}(z)=0 .
$$

By Lemma 1, all the roots of $g(z)=w_{n}$ do lie on a certain straight line of the complex plane (each of which is parallel to the real axis) for $n=1,2, \cdots$. There must exist infinitely many (hence at least four), distinct, straight lines among them. Otherwise, by a theorem of Edrei ([2]), $g$ must reduce to a quadratic polynomial, which is not in reason. Thus, by Kobayashi's theorem ([5]), we have (cf. [8]) that

$$
g(z)=P(\exp [A z]),
$$

where $P$ is a quadratic polynomial and $A$ is a non-zero constant. Since $F_{a}(z)$ $=\sin z \cdot\{h(\cos z)-a \cos z\}$ is an odd function, we have $f\left(P\left(e^{A z}\right)\right)=-f\left(P\left(e^{-A z}\right)\right)$. This implies that $f(P(w))=-f(P(1 / w))$ for $w \neq 0$, from which, comparing the singularity at $w=0$, we get a contradiction. Hence the case a) cannot occur.

b). $f$ is transcendental meromorphic (not entire) and $g$ is transcendental entire. In this case,

and

$$
f(z)=p(z) /\left(z-a_{0}\right)^{k} \quad\left(p\left(a_{0}\right) \neq 0\right)
$$

$$
g(z)=a_{0}+e^{q(z)},
$$

where $k$ is a natural number, $p(z)$ (trans.) and $q(z)$ are entire functions. Then

$$
\begin{aligned}
f(g(z)) & =e^{-k q(z)} \cdot p\left(a_{0}+e^{q(z)}\right) \\
& =\left(e^{-k w} \cdot p\left(a_{0}+e^{w}\right)\right) \circ(q(z)) .
\end{aligned}
$$

If $q$ is transcendental, this case is reduced to the case a). If $q$ is a polynomial, then $\operatorname{deg} q=1$ or 2 by Rényi's theorem $([10])$, since $F_{a}(z)=f(g(z))$ is periodic. When $\operatorname{deg} q=1$ and $q(z)=a z+b$ (say), then putting $\Phi(w)=e^{-k w} \cdot p\left(a_{0}+e^{w}\right)$, we have $\Phi(-a z+b)=-\Phi(a z+b)$, since $F_{a}(z)$ is an odd function. Hence we have $p\left(a_{0}+e^{-a z+b}\right)=-e^{-2 k a z} \cdot p\left(a_{0}+e^{a z+b}\right)$ so that, changing the variable such as $w=e^{a z}$,

$$
p\left(a_{0}+e^{b} / w\right)=-(1 / w)^{2 k} \cdot p\left(a_{0}+e^{b} w\right) .
$$

Comparing the singurality at $w=0$, we get a contradiction (since $p$ is transcendental).

Next, when $\operatorname{deg} q=2$, this case can be reduced to the case c). 
c). $f$ is transcendental entire and $g$ is a polynomial of degree at least two. By Rényi's theorem ([10]), $g$ is a quadratic polynomial. Put $g(z)=s(z-u)^{2}+v$ with constants $s, u$ and $v$. Let $\left\{w_{m}\right\}(m \in \boldsymbol{N})$ be the zeros of $f^{\prime}(w)$. Let $p_{m}$ and $q_{m}$ be two roots of $g(z)=w_{m}$. Then $p_{m}$ and $q_{m}$ are also common roots of the simultaneous equations

$$
F_{a}(z)=f\left(w_{m}\right) \text { and } \quad F_{a}^{\prime}(z)=0 .
$$

Therefore, $\cos p_{m}=\cos q_{m}$ and $\sin p_{m}=\sin q_{m}$ by Lemma 1 (since $a \in E_{0}$ ). Hence, $p_{m}-q_{m}=2 k \pi$ for some integer $k$ and also $\left(p_{m}+q_{m}\right) / 2=u$. Accordingly, $\operatorname{Im} p_{m}$ $=\operatorname{Im} q_{m}=\operatorname{Im} u$. Hence, noting that $F_{a}{ }^{\prime}(z)$ is periodic (with period $2 \pi$ ), we have

This contradicts $(*)$.

$$
\begin{aligned}
N\left(r, 0, F_{a}{ }^{\prime}\right. & \leqq N^{k}\left(r, 0, f^{\prime}(g)\right)+N\left(r, 0, g^{\prime}\right) \\
& =O(r)+O(\log r)=o\left(m\left(r, H^{\prime}\right)\right) .
\end{aligned}
$$

d). $f$ is a polynomial of degree $d(\geqq 2)$ and $g$ is transcendental entire. By Rényi's theorem ([10]), $g$ is periodic. Hence we may put

$$
g(z)=k(\exp [A z]),
$$

where $k(w)$ is holomorphic in $0<|w|<\infty$ and $A$ is a constant $(\neq 0)$. It is noted that $k(w)$ has an essential singularity at $w=0$ or $w=\infty$. Let $x$ be a zero of $f^{\prime}$. Then $k(w)=x$ has at most finitely many roots. Otherwise, the set of the roots of the simultaneous equations $F_{a}(z)=f(x)$ and $F_{a}{ }^{\prime}(z)=0$ (which are distributed periodically on a single straight line, as before, by Lemma 1) has an accumulation point, which is not in reason. Hence, $f^{\prime}$ has exactly one zero, say $x$. Hence

$$
f^{\prime}(w)=b(w-x)^{d-1} \text { and } f(w)=b d^{-1}(w-x)^{d}+c
$$

for some constants $b(\neq 0)$ and $c$. Therefore

$$
F_{a}(z)=b d^{-1}(g(z)-x)^{d}+c .
$$

Hence $N\left(r, c, F_{a}\right)=d N(r, x, g)$. Since $k(w)=x$ has at most finitely many roots, $N(r, x, g)=O(r)=o(m(r, H))$. Thus we have $N\left(r, c, F_{a}\right)=o(m(r, H))$, which is contrary to $(* *)$.

e). $f$ is rational (not a polynomial) and $g$ is transcendental entire. Then

$$
\begin{aligned}
& f(w)=P(w) /\left(w-w_{0}\right)^{k} \quad\left(P\left(w_{0}\right) \neq 0\right), \\
& g(z)=w_{0}+e^{G(z),}
\end{aligned}
$$

where $P$ is a polynomial, $G$ is entire ( $\neq$ const.) and $k$ is a positive integer. Hence

$$
\begin{aligned}
f(g(z)) & =e^{-k G(z)} \cdot P\left(w_{0}+e^{G(z)}\right) \\
& =\left(e^{-k w} \cdot P\left(w_{0}+e^{w}\right)\right) \cdot(G(z)) .
\end{aligned}
$$


Let us note that the function $e^{-k w} \cdot P\left(w_{0}+e^{w}\right)$ is obviously transcendental by the condition $P\left(w_{0}\right) \neq 0$. Hence, this case can be reduced to the case a) or $\mathbf{c}$ ), if $G$ is non-linear. However, if $G$ is linear, the above equation shows that $F_{a}(z)=f(g(z))$ is of order 1 (indeed, of exponential type), which is not in reason. (In this case, $G$ is also transcendental.)

f). $f$ is rational (not a polynomial) and $g$ is transcendental meromorphic (not entire). Let $f\left(w_{0}\right)=\infty$ for some $w_{0} \neq \infty$. Then $g(z)=w_{0}+1 / L(z) \cdot \exp [G(z)]$ for some entire function $L$ and $G$. If we set $g_{1}(z)=1 /\left(g(z)-w_{0}\right)=L(z) \cdot e^{-G(z)}$ (trans. entire) and $f_{1}(\zeta)=f\left(w_{0}+1 / \zeta\right)$ (rational), then we have $f_{1}\left(g_{1}(z)\right)=f(g(z))$. Hence, we see that this case can be reduced to the case d) or e).

Consequently, we get a contradiction (under $F_{a}(z)=f\left(g(z)\right.$ ) with $a \in E_{0}$ ), unless $f$ or $g$ is linear. Hence $F_{a}(z)$ is prime. Thus, the proof of Theorem 1 is now complete.

Proof of Theorem 2 (an outline). Concerning $F_{a}(z)=H(z)(\cos z-a)$, we choose a countable set $E$ of complex numbers for which the assertion of Lemma 2 holds. Now, by the assumption, the order of $H$ and hence that of $F_{a}$ is finite and non-integral. Noting that $F_{a}$ and $F_{a}{ }^{\prime}$ have the same order, we have

$$
\limsup _{r \rightarrow \infty} N\left(r, 0, F_{a}{ }^{\prime}\right) / r=\infty
$$

and

$$
\limsup _{r \rightarrow \infty} N\left(r, c, F_{a}\right) / r=\infty
$$

for any complex number $c$ (in view of Borel's theorem, cf. [6] p. 72). Then, if $a \in E_{0}=C-E, F_{a}(z)$ can be shown to be prime. Assume $F_{a}(z)=f(g(z))$. As in the proof of Theorem 1 , we need to consider the several cases $a) \sim f$ ). In the case a), where $f$ and $g$ are transcendental entire functions, it is noted that $f$ is of order zero (by Pólya's lemma [9]) and hence $f^{\prime}(z)$ has infinitely many zeros. Hence, we can derive a contradiction, by using the similar argument (since $F_{a}(z)$ is also an odd function). Moreover, other cases can be treated quite similarly as before, by applying (\#) or (\#\#) (instead of (*) or (**)). Anyway, we get a contradiction, unless $f$ or $g$ is linear. Therefore, $F_{a}(z)\left(a \in E_{0}\right)$ is prime, which is to be proved.

Acknowledgement. Finally, the author would like to express his gratitude to the referee for valuable comments and kind suggestions.

\section{REFERENCES}

[1] J. Clunie, The composition of entire and meromorphic functions, Mathematical Essays dedicated to A. J. Macintyre (Ohio Univ. Press, 1970), 75-92.

[2] A. EDREI, Meromorphic functions with three radially distributed values, Trans. Amer. Math. Soc. 78 (1955), 276-293. 
[3] F. Gross, Factorization of meromorphic functions, U.S. Government Printing Office, 1972.

[4] W.K. Hayman, Meromorphic functions, Clarendon Press (Oxford), 1964.

[5] T. KobAYASHI, On a characteristic property of the exponential function, Kodai Math. Sem. Rep. 29 (1977), 130-156.

[6] R. Nevanlinna, Le théorème de Picard-Borel et la théorie des fonctions méromorphes, Gauthier Villars (Paris), 1929.

[7] Y. NodA, On factorization of entire functions, Kodai Math. J. 4 (1981), 480-494.

[8] M. Ozawa, On the existence of prime periodic entire functions, Kodai Math. Sem. Rep. 29 (1978), 308-321.

[9] G. Pólya, On an integral function of an integral function, J. London Math. Soc. 1 (1926), 12-15.

[10] A. RENYI AND C. RÉNYI, Some remarks on periodic entire functions, J. d'analyse Math. 14 (1965), 303-310.

[11] G. Song AND J. HuANG, On pseudo-primality of the $n$-th power of prime entire functions, Kodai Math. J. 10 (1987), 42-48.

[12] G. SONG AND L. LIAO, Existence of prime entire function of finite order whose even powers are not pseudo-prime, J. of East China Normal Univ. (Natural Science, 1988, No. 2), 3-9.

Department of Mathematics

Kyoto University of Education

FUSHIMI-KU, KYOTO, 612, JAPAN 